\title{
Editorial
}

\section{Special issue on sickle cell disease: Old and new concepts}

\author{
Philippe Connes ${ }^{\mathrm{a}, \mathrm{b}, \mathrm{c}, *}$ \\ a Laboratoire LIBM EA7424, Team "Vascular Biology and Red Blood Cell”, Université Claude \\ Bernard Lyon 1, University of Lyon, Villeurbanne, France \\ ${ }^{\mathrm{b}}$ Laboratory of Excellence GR-Ex «The red cell: From genesis to death», PRES Sorbonne Paris \\ Cité, Paris, France \\ ${ }^{\mathrm{c}}$ Institut Universitaire de France, Paris, France
}

Sickle cell disease (SCD) is the first molecular disease classified as a molecular disease. The abnormal hemoglobin produced is at the origin of the well-known complications of the disease: i.e., chronic anemia and frequent painful vaso-occlusive crises. SCD is the prototype of a hemorheological disease marked by a severe reduction in red blood cell (RBC) deformability. However, the clinical trajectory of a given patient cannot be predicted from the basic hematological profile and the clinical expression of the disease is highly variable from one patient to another, as well as over time. Indeed, exciting works conducted in the past decades have demonstrated that SCD is more than a hematological disease characterized by the presence of RBC with abnormal shapes.

In this special issue on SCD, Ballas describes the various acute and chronic complications patients may develop, as well as the current (available or under development) strategies and therapies to manage the disease. In another review, Ballas also presents the comorbidities that could develop in aging SCD patients. He discusses the fact that the gain in survival (caused by improved knowledge of the disease and better clinical/therapeutic management) observed in the past decades could be offset by the increased rate of comorbidities classically found in elderly population, and underlines that greater attention will have to be paid to anticipate such situation. The review of Chang et al focused on the major genetic modifiers of SCD clinical severity. Among them, SCD genotype, alpha-thalassemia, UGT1A1 polymorphisms and G6PD deficiency seem to impact the SCD clinical course. The authors also present evidence of the implication of other genetic modifiers in the occurrence of several acute and chronic complications.

The review of Connes and colleagues focused on the role of blood rheology (RBC deformability, RBC aggregation and blood viscosity) in SCD. This is an old topic but recent works demonstrated that its role has been probably sometimes underestimated. More particularly, the authors give evidence that two blood rheological phenotypes would exist: 1) patients with the most rigid RBCs have the highest rate of hemolysis and the most severe anemia and 2) patients having the most deformable RBCs have milder anemia and higher blood viscosity, hence increasing the risks for the occurrence of

\footnotetext{
*Corresponding author: Philippe Connes, PhD, Laboratoire LIBM EA7424, Team "Vascular Biology and Red Blood Cell”, Université Claude Bernard Lyon 1,69100 Villeurbanne, France.E-mails: pconnes@yahoo.fr/philippe.connes@univ-lyon1.fr.
} 
the vaso-occlusive like complications. The review of Detterich discusses the role of blood transfusion and its hemorheological impact in SCD, and presents several new major findings that would stimulate further works to optimize the use of transfusion in this disease. RBC dehydration plays a major role in the pathophysiology of SCD and in the blood rheological modifications usually observed. Brugnara describes the different and major pathways involved in cellular dehydration in SCD: i.e., the K-Cl cotransport, the Gardos channel and Piezo 1 which would be responsible for cellular dehydration through the $\mathrm{P}_{\text {sickle }}$ pathway. The author also summarizes the pharmacological strategies that have been tried to minimize RBC dehydration in SCD.

The large amount of works performed in the field of SCD in the past decades clearly demonstrates that SCD must also be considered as a vascular disease. The review of Usmani and Machado focuses on the vascular part of the disease and more particularly on cerebral, cardiopulmonary and renal vasculopathies. This review with the one of Kim-Shapiro and Gladwin show how hemolysis interferes with the metabolism of nitric oxide (NO), a major vasodilator in human body. They show evidence that hemolysis is responsible for the decrease of NO bioavailability in the vessels, hence participating to the development of endothelial/vascular dysfunction. Kim-Shapiro and Gladwin also proposed new strategies based on the hemolysis-NO paradigm to improve the management of certain SCD complications. In the same way, Van Beers and colleagues demonstrate the key role of reactive oxygen species (ROS) and oxidative stress in SCD pathophysiology and in the development of vasculopathy. More particularly, the authors present the emerging role of the TLR-NOX-ROS axis in SCD. Coates and colleagues present exciting data on the role of the autonomic nervous system in SCD vaso-occlusive crisis. Their recent findings clearly show that vascular dysfunction in SCD could also be explained by the presence of dysautonomia and impaired ANS reactivity to different stressful situations. Higher ANS-mediated vasoconstriction reflex would be involved in the initiation of VOC.

$\mathrm{SCD}$ is also an inflammatory disease characterized by a hypercoagulable state. The impressive review of Conran and Belcher show that sickle RBC, enhanced hemolysis, hemoglobin products and ischemic-reperfusion episodes are responsible for the chronic inflammation observed in SCD and would be responsible for the increased adhesiveness of all circulating cells to the vascular wall, hence participating to the occurrence of VOC. The role of inflammasome, as well as of the gut and micriobiota, in SCD pathophysiology is nicely discussed by the authors. Finaly, the authors list all the anti-inflammatory approaches that could be used in SCD. Faes et al show that several factors are at the origin of the hypercoagulable state of SCD patients, such as increased tissue factor (TF) expression, increased phosphatidylserine (PS) exposure, enhanced thrombin generation and a decrease of natural anti-coagulants, and that hemolysis plays an important role in the activation of coagulation in this disease. Consistent with this chronic hypercoagulable state, the authors present several works showing that SCD patients would be prone to develop thromboembolic complications. Because circulating microparticles (MPs) express a large amount of PS and TF, they are also thought to play a major role in the coagulation process in SCD. Romana et al reviewed the mechanisms at the origin of MPs production, as well as their pathophysiological role, in SCD. MPs are phospholipid microvesicles with a diameter ranging from 100 to $1,000 \mathrm{~nm}$ that are derived from the cytoplasmic membrane of cells submitted to stressful conditions that result in apoptosis or activation. Because RBC-derived MPs would carry a significant amount of heme, they could impair vascular function in SCD and could be involved in the occurrence of VOC. Interestingly the authors discuss the effects of hydroxurea treatment on MPs characteristics.

On the whole, this special issue on SCD reviews recent data showing that although SCD is the first described molecular disease, it is a complicated pathology involving not only abnormal blood rheology but also inflammation, coagulation, oxidative stress, nitric oxide metabolism and endothelial dysfunction. These new findings have stimulated new therapeutical approaches to improve the clinical condition of the patients and should serve in other diseases. 